\title{
Relación entre la aptitud musical y el grado de comprensibilidad del habla en una segunda lengua: estudio de un grupo de alumnos de español de la escuela secundaria inglesa
}

Ángel Osle EzQuerra

Queen Mary, University of London

Recibido: 18 de Enero de 2011 / Aceptado: 7 de Julio de 2011

ISSN: $1697-7467$

\begin{abstract}
RESUMEN: Este artículo explora la relación entre la aptitud musical y el grado de comprensibilidad en una L2. Utilizamos la prueba Bentley para medir la aptitud musical de los sujetos participantes. Asimismo, un grupo de hablantes nativos de español evaluó el grado de comprensibilidad de una serie de muestras orales obtenidas gracias a la descripción de una secuencia fotográfica. Los resultados revelaron una fuerte correlación entre el grado de comprensibilidad y la aptitud musical. Por otro lado, aquellos estudiantes con una formación musical previa obtuvieron mejores resultados tanto en las pruebas de aptitud musical como en las de comprensibilidad del habla.

Palabras clave: Comprensibilidad del habla, aptitud musical, habilidad musical.
\end{abstract}

Exploring the relationship between musical aptitude and degree of comprehensibility in L2 speech: Assessment of a group of English learners of Spanish in the English secondary school system

\begin{abstract}
This article explores the relationship between musical aptitude and degree of comprehensibility in L2 speech. The Bentley test served to measure students' musical aptitude. This test includes sections on pitch discrimination, tonal memory, chord analysis and rhythmic memory. Learners' oral productions were elicited through a picture description task. Furthermore, a group of native Spanish speakers assessed the degree of comprehensibility of students' oral samples. Statistical results revealed a strong correlation between musical aptitude and speech comprehensibility in L2. Students with previous musical training performed significantly better on both musical aptitude tests and degree of comprehensibility of their L2 productions.

Keywords: Musical aptitude, musical ability, L2 speech comprehensibility.
\end{abstract}

\section{InTROduCCIÓN}

Las definiciones relativas al concepto de aptitud musical varían enormemente dependiendo de la perspectiva teórica adoptada. De la misma manera, la terminología utilizada en la literatura engloba conceptos tales como la musicalidad, el talento musical, la habilidad musical o incluso la inteligencia musical. En este artículo, vamos a considerar la 
aptitud musical como «una tendencia innata para el entendimiento/la apreciación, interpretación y creación musical de una manera sobresaliente» (Nardo and Reiterer, 2009: 214). Asimismo, debemos señalar que la evaluación y medición de la aptitud musical es un proceso que no está exento de problemas. Las pruebas más utilizadas para este propósito parecen medir, tal y como señala Shuter-Dyson (1999: 643-645), diversos tipos de habilidades entre las que se incluyen habilidades tonales, rítmicas, kinestésicas, estéticas y creativas. Los instrumentos de medida más utilizados consisten en pruebas psicométricas como el Seashores Measures of Musical Ability, Wings tests of Musical Intelligence, Gordon Measures of Music Audition o Bentley Measures of Musical Abilities. La elección de un instrumento u otro depende, en gran medida, de los objetivos de cada investigador así como del tipo de sujetos participantes en los estudios (véase ShuterDyson and Gabriel, 1981 o Nardo and Reiterer, 2009 para una revisión y descripción detallada de cada una de estas pruebas).

Lo cierto es que existen evidentes similitudes entre la música y el habla: desde semejanzas de tipo estructural hasta ciertas afinidades en cuanto a las zonas del cerebro que se encargan de procesar ambos fenómenos (Isaacs and Trofimovich, 2010: 375). Desde el punto de vista experimental, la relación entre la aptitud musical y la capacidad para adquirir una segunda lengua se viene explorando desde los años 60. Eterno (1961) examinó la posible relación entre la aptitud musical y la corrección en la pronunciación española de un grupo de estudiantes americanos de español. La prueba para medir la aptitud musical (Conn Musical Aptitude Test) incluía una evaluación del ritmo, tempo, tono, melodía y acordes, además de secciones adicionales para medir la capacidad visual y la destreza matemática de los participantes. La prueba de pronunciación utilizaba criterios eminentemente subjetivos y consistía en la lectura en voz alta de 25 palabras. Posteriormente, se determinaba si cada palabra había sido correcta o incorrectamente pronunciada. Eterno concluye que existe una correlación entre aptitud musical y precisión en la pronunciación. Asimismo, afirma que dicha correlación es más intensa a medida que aumenta la edad de los participantes y, en consecuencia, reciben más instrucción en la lengua extranjera.

Leutenegger et al. (1965) llevaron a cabo un estudio con un grupo de estudiantes americanos de francés y español de la Universidad de Florida. La aptitud musical se midió en este caso con el Seashore Test, mientras que la habilidad en la lengua extranjera se determinó como una media del número de errores cometidos por los sujetos en sus pruebas diarias en el laboratorio de idiomas. Posteriormente, se realizaron diferentes comparativas entre los grupos además de utilizar una regresión múltiple para analizar la interacción entre las variables dependientes e independientes. Los resultados revelaron una correlación relativamente débil entre la aptitud musical y la habilidad en la lengua extranjera. Únicamente en el caso de los estudiantes de sexo femenino se pudo apreciar una fuerte correlación entre los resultados de la prueba de memoria tonal y la puntuación obtenida en los test en lengua francesa.

Arellano and Draper (1972) estudiaron la correlación entre la aptitud musical, definida por los resultados obtenidos en cinco de las secciones del Seashore Test, y la habilidad en la producción y comprensión del habla tal y como fue determinada por la puntuación de los participantes en una serie de pruebas de opción múltiple. Los resultados mostraron una fuerte correlación entre entonación, ritmo y pronunciación correcta de ciertos sonidos, así como entre este último aspecto y memoria tonal. 
Brutten et al. (1985) examinaron a un grupo de estudiantes de inglés de diversas lenguas maternas para intentar determinar la relación entre la aptitud musical, la capacidad de discriminación auditiva, y el nivel de competencia de los participantes en lengua inglesa. La aptitud musical fue medida con la prueba Seashore, la capacidad de discriminación auditiva con el Wepman Discrimination Auditory Test y la capacidad de memoria con una prueba de repetición de frases. El nivel de competencia en lengua inglesa se determinó gracias al Test of Spoken English (TSE). Los resultados del estudio mostraron la inexistencia de una correlación entre las pruebas de aptitud musical y los resultados en el TSE.

Más recientemente, Anvari et al. (2002) analizaron las relaciones entre las habilidades de lectura, percepción musical y la conciencia fonológica de un grupo de niños en edad preescolar. Los resultados mostraron una correlación significativa entre las tres dimensiones anteriormente mencionadas. Asimismo, es importante destacar que la percepción musical fue capaz de predecir las habilidades de lectura de los participantes incluso tras la eliminación en el análisis regresivo de la variación compartida con la conciencia fonológica.

En su tesis doctoral, Morgan (2003) estudió la relación entre la percepción y producción musical, y la percepción y producción del habla. Los resultados mostraron una correlación significativa entre percepción musical (ritmo) y percepción del habla. Tal y como sugiere esta autora, los resultados del estudio pueden tener implicaciones para el campo de la enseñanza de lenguas extranjeras ya que los estudiantes dotados de aptitud musical pueden beneficiarse enormemente de un enfoque que enfatice actividades de comprensión oral y ejercicios de percepción musical. Por otro lado, aquellos que carezcan de dicha aptitud podrían utilizar un enfoque de corrección fonética más tradicional.

En una tesis doctoral aún más reciente, Gilleece (2006) examinó la posible relación entre aptitud musical y aptitud para el aprendizaje de segundas lenguas. Se analizó, en concreto, tanto la aptitud receptiva desde un punto de vista musical y lingüístico como la aptitud productiva. Los resultados revelaron una correlación significativa entre aptitud musical y lingüística tanto a nivel receptivo como productivo. Esto último con independencia del grado de inteligencia de los sujetos.

Slevc and Miyake (2006) examinaron la relación entre habilidad musical y nivel de competencia en una segunda lengua en cuatro áreas determinadas: fonología a nivel receptivo y productivo, sintaxis y conocimiento léxico. Los instrumentos de regresión estadística utilizados demostraron la relación entre habilidad musical y habilidad fonológica. Sin embargo, las diferencias en habilidad musical no pudieron predecir la variación en el dominio léxico y sintáctico.

Resultados que avalan la relación entre el concepto de aptitud musical y la habilidad fonética también pueden encontrarse en Nardo and Reiterer (2009). Estos autores utilizaron la prueba de Gordon AMMA para examinar la musicalidad de 66 individuos. El talento para la pronunciación se analizó a través de una prueba específica para evaluar este aspecto en combinación con los tres secciones del MLAT de Carroll and Sapon. Los resultados muestran una correlación alta entre el talento fonético, la sensibilidad gramatical y el grado de musicalidad de los sujetos participantes.

Milovanov et al. (2010) examinaron la posible relación entre aptitud musical, habilidad en la pronunciación de una lengua extranjera y capacidad auditiva para la discri- 
minación fonémica. Los participantes en el estudio fueron un grupo de estudiantes finlandeses de lengua inglesa. La aptitud musical se midió con la prueba Seashore mientras que para las restantes variables se utilizó una prueba de pronunciación y un test de discriminación auditiva. Los resultados no revelaron una correlación entre la aptitud musical y la prueba de discriminación auditiva pero sí que se pudo establecer una relación entre la aptitud musical y los resultados obtenidos por los participantes en la prueba de pronunciación.

También desde un punto de vista neurofisiológico existen estudios que avalan la posible relación entre aptitud musical y habilidad lingüística. En concreto, Wong et al. (2007) proporcionan datos que apuntan a que los individuos con una formación musical exhiben una codificación de patrones lingüísticos de variación de tono mucho más sólida que la de aquellos sujetos sin formación musical alguna. Estudios adicionales que han encontrado evidencias neurológicas a favor de una posible conexión entre aptitud musical y procesamiento lingüístico a nivel fonológico pueden encontrarse en Milovanov et al. (2007, 2008) y Trainor et al. (2009).

La revisión de la literatura llevada a cabo anteriormente parece sugerir que efectivamente existe una relación, tanto a nivel perceptivo como productivo, entre el concepto de aptitud musical y la habilidad para pronunciar una segunda lengua. Esta relación también aparece avalada por experimentos realizados a nivel neurofisiológico aunque debemos señalar que este campo de investigación es relativamente joven y necesitará de un mayor número de estudios para poder llegar a conclusiones más sólidas. Un rasgo característico de todos los estudios mencionados es el uso de diversas pruebas para medir la corrección en la pronunciación de los sujetos participantes. En nuestro caso, vamos a examinar la posible relación entre la aptitud musical y el grado de comprensibilidad del habla en una L2. Este grado de comprensibilidad va a ser entendido como el juicio emitido por un panel de evaluadores sobre la dificultad que entraña la comprensión de las producciones orales de los estudiantes participantes en el estudio. Definir el concepto de comprensibilidad del habla es un trabajo no exento de dificultades. En efecto, la distinción entre comprensibilidad e inteligibilidad no ha sido siempre establecida de manera consistente en la literatura. En el campo de los trastornos del habla, el concepto de inteligibilidad del habla se utiliza de manera frecuente y se refiere normalmente al grado de comprensión que el oyente posee de la señal acústica (Yorkston et al., 1996: 55). Por otro lado, el término comprensibilidad no es tan utilizado ya que el concepto de inteligibilidad se ha extendido para incluir el análisis de variables que no dependen forzosamente de la señal acústica (Yorksdton et al., 1996). Excepciones importantes a este tipo de uso se pueden encontrar en Johnston (2009) o Visser (2004). Estos autores examinan las limitaciones de la medición de la inteligibilidad en el campo de los trastornos del habla y defienden el uso de la comprensibilidad del habla como un término que incluiría información complementaria independiente de la señal acústica (Johnston, 2009: 9). En el campo de la adquisición de segundas lenguas, tanto la inteligibilidad como la comprensibilidad han sido utilizadas de manera inconsistente. Rogers (1997: 2) establece una distinción entre inteligibilidad, entendida como «el grado de éxito logrado por un hablante en el proceso de comunicación y estaría relacionado principalmente con la producción, transmisión o percepción que un oyente realiza de los sonidos de una lengua», y comprensibilidad, término que también aludiría al éxito logrado en el proceso de comu- 
nicación pero en el que se incluirían variables lingüísticas adicionales. Asimismo, el término eficacia comunicativa incluiría variables lingüísticas (factores semánticos o sintácticos) y no lingüísticas. Otros autores como Gass and Varonis (1984) parecen utilizar el término comprensibilidad para referirse a la eficacia comunicativa sin especificar el posible impacto de las distintas variables lingüísticas y no lingüísticas. Nosotros adoptaremos en este artículo la distinción entre inteligibilidad, comprensibilidad y grado de acento extranjero establecida por Derwing and Munro (2009: 478-479) para el campo de la adquisición de segundas lenguas. La inteligibilidad se define como «el grado de comprensión que un oyente posee de un determinado enunciado». Comprensibilidad viene definida como «el grado de facilidad o dificultad que un oyente experimenta durante el proceso de comprensión de un determinado enunciado», mientras que el acento extranjero estaría referido a «las desviaciones observadas en los patrones de los sonidos del habla cuando los comparamos con los patrones que podemos encontrar en la variedad local». Derwing and Munro (Ibíd.) consideran que la inteligibilidad puede ser medida a través de muy diversas técnicas: dictado, trascripción de palabras, grado de comprensión de preguntas etc. Ninguno de estos instrumentos es completamente satisfactorio. Asimismo, estos mismos autores (1997 y 2009) señalan que la inteligibilidad no se encuentra necesariamente correlacionada con el grado de acento extranjero. En otras palabras, un fuerte grado de acento extranjero en la L2 no necesariamente conduce a una reducción en la inteligibilidad del habla. Por otro lado, la inteligibilidad y la comprensibilidad son dos dimensiones que sí parecen estar relacionadas.

En el presente estudio se pretende, pues, explorar la relación existente entre la aptitud musical y el grado de comprensibilidad de las producciones orales de un grupo de estudiantes de español de la escuela secundaria inglesa. Las preguntas que van a guiar esta investigación son las siguientes:

¿Existe una relación global entre aptitud musical y grado de comprensibilidad del habla en una L2?

¿Alguna de las áreas examinadas ofrece un índice de correlación más alto con los juicios relativos a la comprensibilidad de las producciones orales de los participantes en el estudio?

¿Ejercen las variables de género y formación musical previa una influencia significativa sobre los resultados?

\section{Metodología}

\subsection{Participantes}

Los individuos seleccionados pertenecían a una escuela secundaria de tamaño medio del condado de Hertfordshire en Inglaterra. La proporción de estudiantes provenientes de minorías étnicas es la mitad de la media nacional. El último informe Ofsted califica a la escuela como «excelente» en las siguientes áreas: efectividad general de la escuela, desarrollo personal, enseñanza y aprendizaje, apoyo a los estudiantes, liderazgo y gestión. El porcentaje de estudiantes con resultados de entre A y C en los exámenes de GCSE ha aumentado de manera constante en los últimos cinco años, alcanzando un 
$75 \%$ en el último año académico. Por lo que respecta a la enseñanza de lenguas extranjeras, el francés es obligatorio para los estudiantes en Key Stage 3 y 4 . El español tiene carácter opcional a partir del año 10 y se enseña en clases con estudiantes que poseen una amplia gama de habilidades.

Un total de 15 estudiantes de español del año 11 participaron en este estudio. Todos ellos completaron un cuestionario preliminar, firmaron el formulario de consentimiento apropiado y recibieron información por escrito relativa al tipo de investigación que se iba a llevar a cabo. Solo consideramos a hablantes nativos ingleses sin problemas de habla o audición que no hubieran pasado periodos prolongados en países de habla hispana y que no hubieran sido expuestos al español durante su infancia. Inicialmente, seleccionamos a 17 participantes que cumplían con las condiciones establecidas para este estudio. Dos de los estudiantes no completaron todas las sesiones de grabación y fueron posteriormente excluidos del estudio. El objetivo del cuestionario preliminar era la recogida de datos sobre los siguientes extremos: sexo, edad, lugar de nacimiento, lengua nativa, lengua nativa del padre y de la madre, lenguas habladas en casa, número de años de estudio de español, estudio previo de otras lenguas extranjeras y existencia de posibles problemas del habla o audición.

Hay que hacer notar que seis de los participantes, cuyos padres eran de origen polaco o hindú, utilizaban en casa de manera habitual dos lenguas.

Tabla 1. Datos de los participantes.

\begin{tabular}{|c|c|c|c|c|}
\hline Estudiante & Edad & Sexo & L1 & Formación musical \\
\hline 1 & 16 & F & Inglés/punjabi & No \\
\hline 2 & 15 & F & Inglés & No \\
\hline 3 & 15 & M & Inglés/punjabi & No \\
\hline 4 & 15 & F & Inglés/polaco & No \\
\hline 5 & 16 & M & Inglés & No \\
\hline 6 & 15 & M & Inglés/polaco & No \\
\hline 7 & 16 & F & Inglés & No \\
\hline 8 & 15 & M & Inglés/polaco & No \\
\hline 9 & 16 & F & Inglés & Sí \\
\hline 10 & 16 & F & Inglés & Sí \\
\hline 11 & 15 & M & Inglés & Sí \\
\hline 12 & 15 & F & Inglés & Sí \\
\hline 13 & 16 & F & Inglés & Sí \\
\hline 14 & 16 & M & Inglés & Inglés \\
\hline 15 & 16 & M & & \\
\hline
\end{tabular}




\subsection{Evaluadores}

Un total de 10 evaluadores (cinco hombres y cinco mujeres), hablantes nativos de español peninsular (Santander), fueron seleccionados para juzgar el grado de comprensibilidad de las producciones orales de los participantes en este estudio. Variables relacionadas con el oyente tales como el grado de familiaridad con un acento extranjero o los conocimientos lingüísticos previos pueden llegar a afectar a el proceso de evaluación de la comprensibilidad o inteligibilidad del habla (Kennedy and Trofimovich, 2008; Derwing and Munro, 2009; Rogers, 1997). Con el objetivo de neutralizar este tipo de efecto, decidimos seleccionar a hablantes nativos de español sin problemas de audición o habla, de edad variada, con la misma proporción de hombres y mujeres y que no tuvieran contacto con hablantes nativos de lengua inglesa o hubieran pasado un periodo de tiempo prolongado en un país de habla inglesa. Todos los evaluadores completaron un cuestionario preliminar que indagaba sobre los siguientes aspectos: edad, sexo, lugar de nacimiento, lengua materna, lengua materna del padre y de la madre, existencia de problemas de habla o audición, profesión, educación, conocimiento de lenguas extranjeras, contacto con hablantes de lengua inglesa y periodos de tiempo prolongados en países de habla inglesa.

La edad media de los individuos finalmente seleccionados era 26,5. En cuanto al nivel de estudios alcanzado, el $35 \%$ poseía una licenciatura universitaria, el $15 \%$ estaba en esos momentos cursando una licenciatura o diplomatura universitaria, el 30\% tenía un título de bachillerato y un $20 \%$ estaba en posesión de diversos cursos de de formación profesional o simplemente había finalizado la EGB. Ninguno de ellos manifestó mantener contacto habitual con hablantes de lengua inglesa. Algunos sí afirmaron haber pasado periodos de tiempo muy breves en el Reino Unido aunque nunca por un tiempo superior a dos semanas. En cuanto a conocimientos de lenguas extranjeras, el 45\% manifestó no hablar ninguna lengua extrajera, el 30\% afirmó tener un conocimiento básico del francés mientras que un $25 \%$ poseía un conocimiento de la lengua inglesa a nivel elemental. Todos aquellos individuos con competencia a nivel intermedio o avanzado en lengua inglesa fueron excluidos de este estudio.

\subsection{Materiales}

\subsubsection{Medición de la aptitud musical}

Utilizamos el test de medición de habilidades musicales de Bentley (1966) para evaluar la aptitud musical de los sujetos participantes. Originariamente, esta prueba fue concebida para medir las destrezas musicales en niños. Este test está dividido en cuatro partes que intentan medir la discriminación de tono, la memoria tonal, el análisis de acordes y la memoria rítmica. Su fiabilidad estadística es del 0,84 .

La sección dedicada al tono requiere que los participantes distingan entre diferentes tonos de sonidos. Cada estímulo consta de dos tonos debiendo los participantes determinar si el segundo es más alto, más bajo o posee el mismo tono que el primero. Esta sección se compone de 20 pares de sonidos. Los intervalos utilizados son los que normalmente se usan en la música occidental: semitonos, tonos completos, tercios, quintos etc. Otros son microintervalos, es decir, diferencias tonales inferiores a un semitono. 
El test de memoria tonal se basa en la idea de que la apreciación de una melodía es imposible sin la habilidad de recordar en detalle los sonidos que han sido escuchados. Durante esta fase del test, se escuchan diez sonidos. La primera parte de cada estímulo está compuesta por una melodía de cinco notas que se repiten posteriormente con la alteración de una de ellas. Los participantes deben determinar cuál ha sido la nota modificada.

El test de acordes consiste en la presentación de diez acordes de dos notas, ocho de tres notas y dos de cuatro notas. Los sujetos deben identificar cuántas notas se han escuchado en cada ocasión. Bentley afirma que la habilidad para analizar los acordes no es fundamental para la melodía aunque sea una habilidad altamente deseable.

El subtest de memoria rítmica consta de diez estímulos de dos sonidos cada uno. La primera parte de cada estímulo está compuesto por una figura rítmica de cuatro pulsos. En la segunda parte se repite la misma secuencia o, alternativamente, se reproduce otra en la que se ha modificado uno de los pulsos. Los participantes deben decidir si se ha producido o no algún cambio. También deberán anotar el pulso de la nota que ha cambiado.

\subsubsection{Medición de la comprensibilidad del habla}

Se entregó a los participantes en este estudio una serie de fotografías que sirvieron como estímulos visuales con la finalidad de obtener una muestra de sus producciones orales. Se pretendía que los estudiantes contaran la historia sugerida por las fotografías de una forma controlada ya que cada estímulo iba acompañado de una serie de frases y vocabulario clave. El diseño de la prueba está disponible en http://www.splloc.soton.ac.uk/ splloc2/lhnt.html y ha sido utilizada con anterioridad en el proyecto SPLLOC. Los participantes emplearon una media de 3 minutos y 10 segundas en completar la prueba. De cada entrevista se seleccionó un extracto de entre 10 y 15 segundos (cada muestra empezaba y terminaba en un periodo sintáctico). Todas las muestras fueron tomadas de los primeros 30 segundos de la entrevista.

Tras la escucha de cada una de las muestras, los evaluadores procedieron a examinar el grado de comprensibilidad de las mismas de acuerdo con una escala numérica (Derwing and Munro, 1997, 2009) de 1 a 9 en donde 1 indicaba la imposibilidad en la comprensión de la muestra por parte del evaluador y 9 la extremada facilidad en la comprensión de las producciones orales.

\subsection{Procedimiento}

La prueba Bentley se administró en una sola sesión. El componente auditivo de la prueba se reprodujo gracias a un aparato reproductor de audio portátil. Los estudiantes completaron durante la prueba la hoja de respuestas correspondiente. Asimismo, hay que señalar que el test se llevó a cabo en el aula de informática de la escuela secundaria en cuestión, un aula que, por otra parte no contaba con aislamiento acústico.

La grabación de las producciones orales de nuestros participantes se llevó a cabo de forma individual con cada uno de ellos. Se utilizó un ordenador portátil equipado con el programa Audacity para grabar las narraciones de los estudiantes así como de un micrófono USB Samsung C01U. 
Las sesiones de evaluación se llevaron a cabo de manera individual con cada uno de los 10 evaluadores. Cada uno de los ficheros de sonido WAV con las producciones orales de los participantes fue reproducido una sola vez. Tras cada una de las audiciones, los evaluadores seleccionaron uno de los valores de la escala numérica de 1 a 9 a la que ya no hemos referido anteriormente.

\section{Análisis de resultados}

La tabla 2 muestra los resultados obtenidos por los estudiantes en las cuatro secciones de la prueba Bentley, así como el resultado global correspondiente a una media combinada de las cuatro partes. Todos los resultados se expresan en porcentajes para facilitar la labor interpretativa. En cuanto a los resultados globales, las estadísticas descriptivas revelan una media de 74,5 , una mediana de 68,7 y una desviación típica de 13,2 .

Tabla 2. Resultados en la prueba Bentley.

\begin{tabular}{|c|c|c|c|c|c|}
\hline Estudiante & $\begin{array}{c}\text { Discriminación } \\
\text { tonal }\end{array}$ & $\begin{array}{c}\text { Memoria } \\
\text { tonal }\end{array}$ & $\begin{array}{c}\text { Análisis de } \\
\text { acordes }\end{array}$ & $\begin{array}{c}\text { Memoria } \\
\text { rítmica }\end{array}$ & $\begin{array}{c}\text { Total } \\
\text { (media) }\end{array}$ \\
\hline 1 & 75 & 80 & 45 & 60 & 65 \\
\hline 2 & 80 & 80 & 55 & 50 & 66.25 \\
\hline 3 & 65 & 70 & 60 & 50 & 61.25 \\
\hline 4 & 70 & 60 & 65 & 40 & 58.75 \\
\hline 5 & 55 & 80 & 65 & 40 & 60 \\
\hline 6 & 80 & 60 & 55 & 80 & 68.75 \\
\hline 7 & 90 & 50 & 50 & 70 & 65 \\
\hline 8 & 60 & 80 & 65 & 60 & 66.25 \\
\hline 9 & 70 & 80 & 60 & 80 & 72.4 \\
\hline 10 & 90 & 90 & 100 & 90 & 92.5 \\
\hline 11 & 90 & 100 & 75 & 70 & 83.75 \\
\hline 12 & 100 & 80 & 70 & 80 & 82.5 \\
\hline 13 & 90 & 90 & 80 & 90 & 85 \\
\hline 14 & 95 & 100 & 100 & 100 & 97.5 \\
\hline 15 & 90 & 100 & 85 & 100 & 93.7 \\
\hline
\end{tabular}


La tabla 3 muestra la información relativa a los juicios de comprensibilidad del habla realizados por nuestros evaluadores. Hay que señalar que el índice Kappa de Cohen de fiabilidad entre evaluadores se estableció en $0,79, \mathrm{p}<0.001$. En cuanto a las estadísticas descriptivas cabe señalar que la media ascendió a 7,03, la mediana a 7 y la desviación típica fue de 1,35 .

Tabla 3. Resultados en la prueba de comprensibilidad.

\begin{tabular}{|c|c|c|c|}
\hline Estudiante & Punctuación media & Media punctuation max & Media puntuación min \\
\hline 1 & 6,50 & 7,10 & 5,25 \\
\hline 2 & 5,87 & 6,55 & 4,60 \\
\hline 3 & 7,00 & 7,50 & 5,90 \\
\hline 4 & 6,55 & 6,90 & 5,32 \\
\hline 5 & 5,32 & 6,10 & 4,18 \\
\hline 6 & 6,75 & 7,25 & 5,50 \\
\hline 7 & 7,10 & 7,35 & 6,05 \\
\hline 8 & 4,95 & 5,20 & 3.75 \\
\hline 9 & 5,05 & 5,12 & 4,00 \\
\hline 10 & 8,85 & 8,90 & 7,90 \\
\hline 11 & 8,65 & 8,75 & 7,48 \\
\hline 12 & 7,95 & 8,12 & 6,88 \\
\hline 13 & 8,05 & 8,25 & 7,02 \\
\hline 14 & 9,00 & 9,13 & 7,95 \\
\hline 15 & 8,00 & 8,16 & 6,88 \\
\hline
\end{tabular}

Utilizamos la prueba $\mathrm{T}$ para determinar si el género de los participantes había contribuido a la producción de diferencias significativas de rendimiento tanto en la prueba de aptitud musical como en la del grado de comprensibilidad del habla. En cuanto al rendimiento de los participantes en la prueba de aptitud musical, los resultados revelan que no existieron diferencias significativas entre individuos del sexo masculino $(M=75,9$, $\mathrm{SD}=15,5)$ y femenino $(\mathrm{M}=73,4, \mathrm{SD}=11,90), \mathrm{t}=(13) 0,347, \mathrm{p}=0.734)$. En cuanto a los resultados relativos al grado de comprensibilidad del habla, tampoco se detectaron diferencias significativas entre ambos grupos: $t=(13) 0,147, p=0,88$.

Por lo que se refiere a las diferencias entre aquellos participantes con o sin formación musical previa, la prueba $\mathrm{T}$ demostró la existencia de diferencias significativas entre ambos grupos tanto por lo que se refiere a la aptitud musical $(t=(13) 8,988, p=0,001)$ como al grado de comprensibilidad del habla $(\mathrm{t}=(13) 6,746, \mathrm{p}=0,001)$.

El objetivo principal de este estudio es examinar la posible relación entre la aptitud musical y el grado de comprensibilidad de las producciones orales de los individuos 
participantes. Con el fin de examinar esta relación, calculamos el coeficiente de correlación de Spearman entre ambas variables. En primer lugar, debemos señalar que optamos por utilizar Spearman rho en lugar de Pearson $r$ debido al reducido número de participantes, a no poder asegurar la normalidad en la distribución de la muestra y a la escalas de puntuación utilizadas en la medición de las diferentes variables. La interpretación de los coeficientes de correlación de Spearman es similar al de Pearson r. Los coeficientes pueden oscilar entre +1 y -1 . Tal y como señala Cohen (1988), un índice de correlación es pequeño cuando se encuentra entre $\ll 0,3$ y «0,1 o de 0.1 a 0.3 , medio de $\ll 0,5$ a « 40,3 o de 0,3 a 0,5 y elevado de $\ll 1.0$ a $\ll 0,5$ o de 0,5 a 1 .

En nuestro estudio calculamos los coeficientes de correlación entre la puntuación total obtenida en la prueba Bentley y los resultados derivados de los juicios de comprensibilidad del habla. Asimismo, también calculamos el coeficiente de correlación entre esta última variable y cada una de las subsecciones de la prueba Bentley. La tabla 4 presenta los coeficientes correlación entre las distintas variables.

Tabla 4. Índices de correlación.

\begin{tabular}{|l|l|r|r|r|r|r|}
\hline & & DiscTon & MemTon & AnAcord & MemRit & Comprens \\
\hline Discrimincion Tonal & Spearman rho & 1.000 & .454 & .478 & $.741^{* *}$ & $.825^{* *}$ \\
\hline & &. & .089 & .072 & .002 & .000 \\
\hline & $\mathrm{N}$ & 15 & 15 & 15 & 15 & 15 \\
\hline MemoriaTonal & Spearman rho & .454 & 1.000 & $.771^{* *}$ & $.594^{*}$ & $.542^{*}$ \\
\hline & & .089 &. & .001 & .019 & .037 \\
\hline & $\mathrm{N}$ & 15 & 15 & 15 & 15 & 15 \\
\hline Analisis Acordes & Spearman rho & .478 & $.771^{* *}$ & 1.000 & $.589^{*}$ & $.672^{* *}$ \\
\hline & & .072 & .001 &. & .021 & .006 \\
\hline & $\mathrm{N}$ & 15 & 15 & 15 & 15 & 15 \\
\hline Memoria Ritmica & Spearman rho & $.741^{* *}$ & $.594^{*}$ & $.589^{*}$ & 1.000 & $.668^{* *}$ \\
\hline & & .002 & .019 & .021 &. & .006 \\
\hline & $\mathrm{N}$ & 15 & 15 & 15 & 15 & 15 \\
\hline Comprensibilidad & Spearman rho & $.825^{* *}$ & $.542^{* *}$ & $.672^{* * *}$ & $.668^{* * *}$ & 1.000 \\
\hline & & .000 & .037 & .006 & .006 &. \\
\hline & $\mathrm{N}$ & 15 & 15 & 15 & 15 & 15 \\
\hline
\end{tabular}

**Correlación significativa para $\mathrm{p}=0,01$

*Correlación significativa para $\mathrm{p}=0,05$

Como puede apreciarse, existe una correlación significativamente elevada entre la variable de comprensibilidad y cada una de los subtest incluidos en la prueba Bentley. El índice de correlación es particularmente elevado entre el grado de comprensibilidad y la discriminación tonal. También se aprecia una fuerte correlación entre la memoria rítmica, el análisis de acordes y la comprensibilidad del habla. El coeficiente de correlación es moderado entre la memoria tonal y la comprensibilidad. Hay que hacer notar igualmente la significativa correlación que se puede apreciar entre algunas de las secciones de la prueba Bentley. Esto último es algo que esperábamos ya que los diferentes componentes 
de esta prueba miden aspectos relacionados con la aptitud musical y, por tanto, puede considerarse como lógico un cierto grado de solapamiento entre los mismos.

\section{Conclusiones}

Los resultados analizados en el apartado anterior nos permiten responder, con cierta cautela, a las preguntas planteadas al inicio de nuestro estudio. En primer lugar, parece existir una correlación significativa entre el grado de comprensibilidad de las producciones de los participantes en una L2 y su aptitud musical. Se confirman pues los resultados obtenidos en estudios anteriores (véase nuestra sección introductoria) en los que se habían establecido conexiones entre la aptitud musical y la facilidad para el aprendizaje de diversos aspectos de una lengua extranjera. Hay que subrayar el hecho de que, en nuestro caso, la relación se establece entre los resultados logrados con un instrumento específico de medición de la habilidad musical, la prueba Bentley, y los juicios que sobre comprensibilidad del habla han sido emitidos por nuestro grupo de evaluadores.

Al mismo tiempo, las diferencias de género no parecen ejercer una influencia significativa sobre los resultados. Igualmente, hay que señalar que el grupo de participantes con formación musical previa ha obtenido un grado de comprensibilidad del habla significativamente mayor que el grupo de alumnos sin formación alguna. En efecto, estudios anteriores (Douglas and Willatts, 1994) han demostrado que la formación musical previa puede tener efectos positivos sobre ciertos aspectos de la adquisición de una segunda lengua. Al mismo tiempo, esta misma variable ha ejercido una influencia significativa sobre los resultados obtenidos por nuestros participantes en la prueba Bentley. En otras palabras, la aptitud musical puede ser una variable que interactúe con la formación musical y, por consiguiente, es de esperar que aquellos sujetos con mayor aptitud musical se beneficien en mayor medida de seguir una formación musical.

En cuanto a las limitaciones a este estudio, hay que subrayar el hecho de que la muestra empleada es pequeña y esto puede ocasionar ciertas distorsiones a la hora de analizar los datos estadísticos. Asimismo, es evidente que podemos criticar la idoneidad de ciertos aspectos de la prueba Bentley. Igualmente, hay que reconocer que pueden haber existido variables, que no han sido tenidas en consideración, y que hayan ejercido una influencia sobre los juicios realizados por nuestros evaluadores.

Como hemos vista en la revisión de la literatura realizada al inicio, los estudios que analizan la relación entre la aptitud musical y la comprensibilidad del habla en una L2 son más bien escasos. Como sugerencia para el futuro, apuntamos la posibilidad de examinar la relación entre el concepto de aptitud musical y dimensiones del habla tales como la inteligibilidad o el grado de acento extranjero. Asimismo, sería necesario explorar cómo los diferentes niveles de competencia interactúan con la aptitud musical y las distintas dimensiones del habla en una segunda lengua. Desde el punto de vista de las posibles aplicaciones didácticas, el desarrollo de enfoques que incluyan o potencien la utilización de elementos musicales podría ser adecuado especialmente para aquellos alumnos que poseen un mayor grado de aptitud musical. En esta línea, creemos que sería necesaria la exploración de la efectividad de una instrucción formal basada en la introducción de tales elementos con estudiantes de distintos niveles de competencia lingüística. 


\section{Bibliografía}

Anvari, S. H., Trainor, L. J., Woodside, J. and Levy, B. A. (2002). «Relations among musical skills, phonological processing, and early reading ability in preschool children». J Exp Child Psychol, 83(2): 111-30.

Arellano, S. I. and Draper, J. E.(1972). «Relations between musical aptitudes and second-language learning». Hispania, 55: 111-21.

Bentley, A. (1966). Measures of music abilities. Berkshire: NFER-Nelson Publishing Company.

Brutten, S. R., Angelis, P. J., and Perkins, K. (1985). «Music and memory: Predictors for attained ESL oral proficiency». Language learning, 35: 299-313.

Cohen, J. (1988). Statistical power analysis for the behavioral sciences ( $2^{\text {nd }}$ edition). New Jersey: Lawrence Erlbaum.

Derwing, T.M. and Munro, M.J. (1997). «Accent, intelligibility, and comprehensibility: Evidence from four L1s». Studies in Second Language Acquisition, 19(01), 1-16.

Derwing, T.M. and Munro, M.J., (2009). «Putting Accent in Its Place: Rethinking Obstacles to Communication». Language Teaching, 42(4), 476-490.

Douglas, S., and Willatts, P. (1994). «The relationship between musical ability and literacy skills». Journal of Research in Reading, 17(2), 99 - 107.

Eterno, J. A.(1961). «Foreign language pronunciation and musical aptitude». Modern Language Journal, 45: 168-170.

Gass, S. and Varonis, E., (1984). «The effect of familiarity on the comprehensibility of nonnative speech». Language Learning, 34, 65-89.

Gilleece, L. F. (2006). An empirical investigation of the association between musical aptitude and foreign language aptitude. Doctoral Thesis, University of Dublin, Trinity College.

Isaacs, T., and Trofimovich, P. (2010). «Falling on sensitive ears? The influence of musical ability on extreme raters' judgments of L2 pronunciation». TESOL Quarterly, 44, 375-386.

Johnston, J. (2009). Comprehensibility assessment. The influence of familiar and unfamiliar communication partners. Unpublished master's thesis, University of British Columbia, Vancouver, British Columbia, Canada.

Kennedy, S. and Trofimovich, P. (2008). «Intelligibility, comprehensibility, and accentedness of L2 speech: the role of listener experience and semantic context». Canadian Modern Language Review/La Revue canadienne des langues vivantes, 64(3), 459-489.

Leutenegger, R. R., Mueller, T. H., and Wershow, I. R. (1965). «Auditory factors in foreign language acquisition». Modern Language Journal, 49: 22-31.

Milovanov, R., Tervaniemi, M., Takio, F., and Hämäläinen, H. (2007). «Modification of dichotic listening (DL) performance by musico-linguistic abilities and age». Brain Research, 1156: $168-173$.

Milovanov, R., Huotilainen, M., Välimäki, V., Esque, P. A., and Tervaniemi, M. (2008). «Musical aptitude and second language pronunciation skills in school-aged children: Neural and behavioral evidence». Brain Research, 1194: 81-89.

Milovanov, R., Pietilä, P., Tervaniemi, M., and Esquef, P. A. A. (2010). «Foreign language pronunciation skills and musical aptitude: A study of Finnish adults with higher education». Learning and Individual Differences, 20(1), 56-60.

Morgan, C. (2003). Musical aptitude and second-language phonetics learning: implications for teaching methodology. Doctoral Thesis, Simon Fraser University. Music Educators National Conference (MENC). 
Nardo, D. and Reiterer, S. (2009). «Musicality and phonetic language aptitude». In: Dogil, G. and Reiterer, S. (eds.) Language Talent and Brain Activity. Trends in applied linguistics1, Mouton de Gruyter, Berlin-New York.

Rogers, C., (1997). Intelligibility of Chinese-accented English. Unpublished doctoral dissertation. Indiana University.

Shuter-Dyson, R. and Gabriel, C. (1981). The psychology of musical ability. London: Methuen.

Shuter-Dyson, R. (1999). «Musical ability». In Deutsch D. (Ed.) The psychology of music (second edition). London: Academic Press.

Slevc, L. R. and Myiake, A.( 2006). «Individual differences in second-language proficiency. Does musical ability matter?». Psychological Science, 17(8): 675-681.

Trainor, L. J., Shahin, A., and Roberts, L. E. (2009). «Effects of musical training on the auditory cortex in children». Annals of the New York Academy of Sciences, 999, 506-513.

Visser, T. (2004). Comprehensibility: A potential measure for improvement in communication. Unpublished master's thesis, University of British Columbia, Vancouver, British Columbia, Canada.

Wong, P. C. M., Skoe, E., Russo, N. M., Dees, T. and Kraus, N. (2007). «Musical experience shapes human brainstem encoding of linguistic pitch patterns». Nature Neuroscience, 10, 420-422.

Yorkston, K. M., Strand, E. A., and Kennedy, M. R. (1996). «Comprehensibility of dysarthric speech: Implications for assessment and treatment planning». American Journal of SpeechLanguage Pathology, 5, 55-66. 